\title{
Tratamiento de la Hipertensión Gestacional con Endralazina
}

\author{
Drs : Soto Yances Antonio*, Hernández Arias Víctor**, Vargas Moreno Raúl*** \\ Niebles Rafael****, Barrios Amaya Jaime*****
}

\section{Introducción}

La hipertensión arterial durante el embarazo constituye una de las causas más importantes de morbilidad y mortalidad materna y perinatal y plantean al médico un problema sumamente difícil en su manejo y tratamiento.

Diversos autores han demostrado que el diagnóstico y tratamiento temprano y adecuado de la hipertensión puede reducir significativamente la morbi-mortalidad. Cabe señalar que la hipertensión puede ser de corta o larga duración, previa al embarazo o aparecer por vez primera durante el mismo.

Las opiniones difieren acerca del valor del tratamiento $y$ de las drogas utilizadas, así como del efecto sobre el feto $y$ el recién nacido.

\footnotetext{
* Profesor Titular. Departamento de Obstetricia y Ginecología.

* Instructor Asociado. Departamento de Obstetricia y Ginecología.

*** Instructor Asociado. Departamento de Ginecología y Obstetricia.

**** Instructor Asociado. Departamento de Medicina Interna.

***** Jefe. Departamento de Obstetricia y Ginecología.
}

El presente estudio tiene como objetivo demostrar el efecto antihipertensor de la Endralazina (Miretilan-Sandoz) en la hipertensión gestacional; así como las posibles repercusiones sobre los productos del metabolismo nitrogenado en sangre materna y el estado del recién nacido.

\section{Material y Métodos:}

El estudio comprende 20 pacientes embarazadas, previamente diagnosticadas como hipertensas gravídicas (no toxémicas), con edad gestacional mayor de 28 semanas (tercer trimestre) y presión diastólica mayor de $100 \mathrm{mmHg}$, a las cuales se administró endralazina durante 4 semanas, en dosis progresivas de 5 a $30 \mathrm{mg}$ diarios, de acuerdo al siguiente esquema terapéutico.

La presión arterial se tomó con un tensiómetro calibrado siguiendo la metodología establecida por la "American Heart Association", registrando como valor definitivo la media de por lo menos 3 lecturas en posición erecto $y$ decúbito dorsal. En todas las pacientes se dosificaron los productos del metabolismo nitrogenado en sangre materna y el estado del recién nacido se evaluó por el puntaje de Apgar al 10. y 5o. minuto del nacimiento. 
Cuadro No. 1

\begin{tabular}{|l|c|c|c|c|c|c|}
\hline Semana & -2 & -1 & 1 & 2 & 3 & 4 \\
\hline Dósis & 0 & & $\begin{array}{c}2.5 \\
\text { BID }\end{array}$ & $\begin{array}{c}2.5 \\
\text { TID }\end{array}$ & $\begin{array}{c}5 \\
\text { TID }\end{array}$ & $\begin{array}{c}10 \\
\text { TID }\end{array}$ \\
\hline
\end{tabular}

\section{Resultados}

Presión arterial: $\mathrm{Al}$ iniciar el tratamiento fue de $185 / 120+5.0 / 3.0$ $\mathrm{mmHg}$ en posición decúbito dorsal y de $170 / 125 \pm 4.0 / 3.0$ en erecto $y$ al final del mismo, con dosis de $10 \mathrm{mg}$ TID, se observó una significativa disminución en las cifras tensionales, a niveles de 140/90 $\pm 3.0 / 2.0$ en decúbito dorsal y $145 / 95 \pm$ 3.0/2.0 en erecto, haciendo notar que esta reducción se manifestó en forma progresiva de acuerdo a la dosis administrada, lográndose estabilizar y mantener con $10 \mathrm{mg}$ TID.

Pulso Materno: Al iniciar el tratamiento fue de $84 \pm 6$ latidos por minuto en decúbito dorsal y de $92 \pm 4$ latidos por minuto en erecto, y al final del mismo se observó una significativa disminución a valores de $76 \pm 2$ en decúbito dorsal y $84 \pm 2$ latidos por minuto en erecto, cuando la droga se administró a dosis de $10 \mathrm{mg}$ TID, efecto terapéutico que se manifestó en forma progresiva y relacionada a la disminución de la presión arterial (Cuadro No. 2).

\section{Cuadro No. 2}

$N: 20 \quad P: \leqslant 0.001$

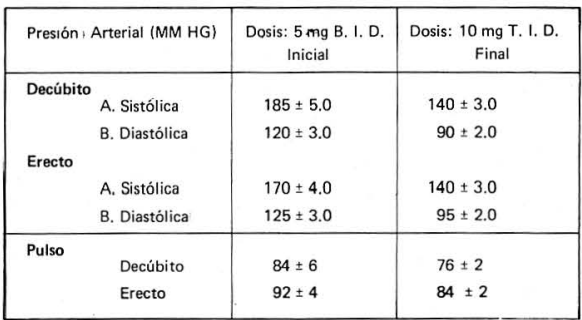

Exámenes de laboratorio: La dosificación sistemática de los productos del metabolismo nitrogenado en sangre materna, tales como creatinina nitrógeno uréico y ácido úrico, demostraron una disminución significativa de los valores hallados al final del tratamiento con relación a los niveles iniciales.

Los valores consignados en el cuadro anterior permiten establecer que la reducción de estos metabolitos es progresiva y acorde con la dosis de Endralazina, obteniéndose cifras normales al final del tratamiento a dosis de $10 \mathrm{mg}$ TID.

Cuadro No. 3

\begin{tabular}{|l|c|c|c|c|c|c|}
\hline \multirow{2}{*}{$\begin{array}{c}\text { Bioqu ímica Sanguínea materna } \\
\text { Mgs \% }\end{array}$} & \multicolumn{3}{|c|}{ Inicial } & \multicolumn{3}{c|}{ Final } \\
\cline { 2 - 7 } & Media & D.S. & Rango & Media & D S & Rango \\
\hline Creatinina & 2.42 & +0.26 & 3.6 & 2.01 & +0.19 & 2.8 \\
& & & 1.8 & & & 1.2 \\
\hline Nitrógeno Uréico & 22.1 & \pm 1.4 & 27.8 & 16.6 & +0.8 & 19.8 \\
& & & 15.4 & & & 11.0 \\
\hline Acido Urico & 7.1 & \pm 0.5 & $\begin{array}{c}11.0 \\
4.8\end{array}$ & 5.6 & \pm 0.3 & 7.2 \\
& & & & & 5.2 \\
\hline
\end{tabular}


Tipo de parto: Del grupo de 20 pacientes estudiadas, el embarazo terminó en operación cesárea antes de las 38 semanas en 5 de ellas y en parto vaginal espontáneo o inducido en las 16 restantes (Cuadro No. 4).

\section{Cuadro No. 4}

N: 20

\begin{tabular}{|l|c|c|}
\hline \multirow{2}{*}{ Tipo de Parto } & \multicolumn{2}{|c|}{ Edad Gestacional } \\
\cline { 2 - 3 } & $\leqslant 38$ semanas & $\geqslant 38$ Semanas \\
\hline Cesárea & $5(25 \%)$ & - \\
\hline Parto Vaginal & $3(15 \%)$ & $12(60 \%)$ \\
\hline Totales & $8(40 \%)$ & $12(60 \%)$ \\
\hline
\end{tabular}

La causa de la cesárea fue en su mayor ía debida a insuficiencia placentaria, demostrada básicamente por el test de Pose (Prueba de tolerancia fetal a las contracciones uterina inducidas).

La evaluación del recién nacido: Se hizo mediante el puntaje de Apgar al 10. y 5o. minuto del nacimiento, registrándose los siguientes datos:

Cuadro No. 5

N: 20

\begin{tabular}{|c|c|c|c|}
\hline \multirow{2}{*}{ Estado del Recién-Nacido } & \multicolumn{2}{|c|}{ Puntaje de Apgar } & \multirow{2}{*}{ Total } \\
\hline & $1 \mathrm{A7}$ & $7 A 10$ & \\
\hline Vivo & $4(22 \%)$ & $14(68 \%)$ & $18(90 \%)$ \\
\hline $\begin{array}{l}\text { Muerte Fetal } \\
\text { (Intrauterina) }\end{array}$ & - & - & $2(10 \%)$ \\
\hline & & & $20(100 \%)$ \\
\hline
\end{tabular}

Los dos casos de muerte fetal intrauterina se comprobó fue debida a severa insuficiencia placentaria en pacientes que desarrollaron una crisis hipertensiva a las 33 y 35 semanas de gestación respectivamente.

\section{Conclusiones}

Los resultados anteriores permiten afirmar que la Endralazina (MiretilanSandoz) posee un significativo efecto antihipertensor en pacientes con hipertensión gravídica y que esta acción es progresiva de acuerdo a la dosis $y$ al tiempo de administración, obteniéndose la estabilización de la presión arterial a dosis de $10 \mathrm{mg}$ TID.

Igualmente se observa una reducción progresiva en los valores de los productos del metabolismo nitrogenado (creatinina - nitrogeno uréico y ácido úrico), logrando obtener cifras normales al final del tratamiento, hecho que está directamente relacionado a la disminución de la presión arterial $y$ del pulso materno.

La evaluación del recién nacido, mediante el puntaje de Apgar, no mostró clínicamente cambios significativos.

\section{Descripción Farmacológica}

La Endralazina es un vasodilatador periférico que posee una potente acción hipotensora. Los estudios clínicos han demostrado que el efecto hipotensor es dosis dependiente $y$ que induce taquicardia, aún a bajas dosis. La administración simultánea de betabloqueadores previene estos efectos y potencializa su acción hipotensora, aumentando así su utilidad clínica.

La dosis de Endralazina requerida para reducir la presión arterial varía considerablemente de paciente por paciente, pero su efecto terapéutico aumenta progresivamente con la dosis y el tiempo de administración.

\section{Resumen}

Se presenta 20 casos con diagnóstico confirmado de hipertensión gravídica 
(no toxémicas), con edad gestacional mayor de 28 semanas y presión diastólica mayor de $100 \mathrm{mg} \mathrm{Hg}$, a las cuales se administró Endralazina (Miretilan-Sandoz) durante 4 semanas, en dosis progresivas de 5 a $30 \mathrm{mg}$ diarios, observándose una disminución igualmente progresiva de la presión arterial de acuerdo a la dosis $y$ al tiempo de administración, lográndose estabilizar con $10 \mathrm{mg}$ TID.
Igualmente, se dosificaron los productos del metabolismo nitrogenado en sangre materna (creatinina, nitrógeno uréico y ácido úrico), notándose una reducción progresiva de los mismos hasta llegar a valores normales al final del tratamiento.

Se evaluó el estado del recién-nacido por el puntaje de Apgar, no observándose cambios clínicos significativos.

\title{
TREATMENT OF GESTATIONAL HYPERTENSION WITH ENDRALAZINE
}

\author{
By Soto-Yances A. et al. Universidad de Cartagena - Colombia
}

\section{Summary}

This article analyzes 20 cases of hypertension due to gravidity (not toxemias), with a gestational age of more than 28 weeks and a diastolic blood pressure of more than $100 \mathrm{~mm}$. of mercury. Clinicians administered Endralazine (Miretilan-Sandoz) during four weeks, progressively increasing the dosage from $5 \mathrm{mg}$. to $30 \mathrm{mg}$. every day. Treatment resulted in a decrease in

\section{Bibliografía}

1. GANT, NORMAN Y COLS. “Embarazo e Hipertensión". Seminarios de Perinatología. Edit. Grune and Stratton. 1980.

2. HOLMES, DAVID. "Miretilan: Results of two years treatment in patients with Hypertension". Medical Research Dpment. Marzo 1982.

3. LEWIS, P.J. Y COLS. Tratamiento de la Hipertensión en la mujer embarazada. "Problemas terapéuticos en el embarazo" Edit. Pediátrica, Barcelona. Págs. 61-72 1978. blood pressure according to the dosage and the moment of administration, reaching blood pressure stability with 10 mg TID.

The substances product of nitrogenated metabolism in the mothers' blood were dosed (creatinine, ureic nitrogen and uric acid), and showed a clear reduction, reaching normal vales by the end of the treatment.

4. POSE, S.V. CASTILLO, J. MORA, E.O. SOTO-YANCES, A Y CALDEYRO-BARCIA. R. "Prueba de Tolerancia Fetal a las contracciones uterinas inducidas". Factores que afectan el Desarrollo Humano. Pụblicación de P.A.H.O. 1969.

5. "Treatment of Hypertension in Pregnan$\mathrm{cy}^{\prime \prime}$. Drug and Therap. Bullettin Vol. 20 No. 1. 1982.

6. WELT, S., CRENSHAW, M. "Embarazo e Hipertension concomitante". Clin. Obstet. Ginec. Vol. 3. Pág. 653-685, 1978.

7. ZACEST, $R$ y KOCH-WESER, J. Clinic Pharm. Therap 13,420-5. 1972. 